TITLE:

\title{
Responses of monkey prefrontal neurons during the execution of transverse patterning
}

\section{$\operatorname{AUTHOR}(\mathrm{S})$ :}

Nejime, Masafumi; Inoue, Masato; Saruwatari, Masanori; Mikami, Akichika; Nakamura, Katsuki; Miyachi, Shigehiro

\section{CITATION:}

Nejime, Masafumi ...[et al]. Responses of monkey prefrontal neurons during the execution of transverse patterning. Behavioural Brain Research 2015, 278: 293-302

\section{ISSUE DATE:}

2015-02

URL:

http://hdl.handle.net/2433/191225

\section{RIGHT:}

(c) 2014 Elsevier B.V.; この論文は著者最終稿です。内容が印刷版と異な ることがありますので、引用の際には出版社版をご確認ご利用くださ い。 This is the Accepted Author Manuscript. Please cite only the published version. 


\section{Title}

Responses of monkey prefrontal neurons during the execution of transverse patterning

\section{Author names and affiliations}

Masafumi NEJIMEa, Masato INOUEa, Masanori SARUWATARIa, Akichika MIKAMIa,b, Katsuki NAKAMURA ${ }^{a}$, Shigehiro MIYACHIa

aCognitive Neuroscience Section, Primate Research Institute, Kyoto University, Inuyama, Aichi 484-8506, Japan, bFaculty of Nursing and Rehabilitation, Chubu Gakuin University, Kirigaoka 2-1, Seki, Gifu 501-3993, Japan

\section{Corresponding author}

Shigehiro MIYACHI

Address: Cognitive Neuroscience Section, Primate Research Institute, Kyoto University, Inuyama, Aichi 484-8506, Japan

E-mail: miyachi.shigehiro.8e@kyoto-u.ac.jp

Tel: +81-568-63-0275 Fax: +81-568-63-0563

\section{Present address}

Masato INOUE

Department of Neurophysiology, Juntendo University Graduate School of Medicine, Hongo 2-1-1, Bunkyo, Tokyo 113-8421, Japan

\section{Masanori SARUWATARI 1,2}

${ }^{1}$ Department of Brain Physiology, Graduate School of Medicine, Osaka University, Yamadaoka 2-2, Suita, Osaka 565-0871, Japan

${ }^{2}$ Center for Information and Neural Networks, National Institute of Information and Communications Technology and Osaka University, Yamadaoka 1-4, Suita, Osaka 565-0871, Japan 


\section{Abbreviations}

ANOVA, analysis of variance; $\mathrm{CgS}$, cingulate sulcus; CRT, cathode ray tube; DC, dorsal convexity; DMS, delayed matching-to-sample; EEG, electroencephalography; fMRI, functional MRI; HSD, honestly significant difference; ICMS, intracortical microstimulations; i.m., intramuscular; MEG, magnetoencephalography; MPF, medial prefrontal cortex; MR, magnetic resonance; MTL, medial temporal lobe; PF, prefrontal cortex; PS, principal sulcus; sAS, superior limb of arcuate sulcus; TP, transverse patterning 


\section{Abstract}

Recent functional imaging studies have suggested that the prefrontal cortex $(\mathrm{PF})$ is engaged in the performance of transverse patterning (TP), which consists of 3 conflicting discriminations (A+/B-, B+/C-, C+/A-). However, the roles of $\mathrm{PF}$ in $\mathrm{TP}$ are still unclear. To address this issue, we examined the neuronal responses in 3 regions [the principal sulcus (PS), dorsal convexity (DC), and medial prefrontal cortex (MPF)] of the macaque PF during the performance of an oculomotor version of TP. A delayed matching-to-sample (DMS) task was used as a control task. The TP task-responsive neurons were most abundant in MPF. We analyzed the dependency of each neuronal response on the task type (TP or DMS), target shape (A, B, or $\mathrm{C}$ ), and target location (left or right). Immediately after the choice cue presentation, many MPF neurons showed task dependency. Interestingly, some of them already exhibited differential activity between the 2 tasks before the choice cue presentation. Immediately before the saccade, the number of target location-dependent neurons increased in MPF and PS. Among them, many MPF neurons were also influenced by the task type, whereas PS neurons tended to show location dependency without task dependency. These results suggest that MPF and PS are involved in the execution of TP: MPF appears to be more important in the target selection based on the TP rule, whereas PS is apparently more related to the response preparation. In addition, some neurons showed a postsaccadic response, which may be related to the feedback mechanism.

\section{Keywords}

Single neuronal activity, Nonhuman primate, Medial prefrontal cortex, Configural discrimination, Nonlinear association, Long-term memory 


\section{Introduction}

Transverse patterning (TP) is a learning paradigm that requires learning a series of visual discrimination tasks with 3 stimuli [1]. In TP, the correct or rewarded item depends on the context. For example, when A and B are paired, A is correct; when B and C are paired B is correct; and when $\mathrm{C}$ and $\mathrm{A}$ are paired, $\mathrm{C}$ is correct. The third discrimination $\mathrm{C}$ from $\mathrm{A}$ is relatively difficult because the discrimination is against a logical inference that $A$ is rewarded over B and B is rewarded over C [2-4]. Thus TP is a kind of nonlinear learning, in which stimuli are configured $[1,5,6]$.

To date, the most important findings on animal responses to TP are that rats and monkeys with bilateral damage to the medial temporal structures including the hippocampus are severely impaired in the third discrimination of TP [7-11]. In addition, amnesic patients with hippocampal damage failed to solve the third discrimination of TP [12,13]. Animals with hippocampal damage could solve some other configural learning tasks, and therefore the role of the hippocampus in configural learning may be circumscribed [5,14-16].

On the other hand, recent brain imaging studies with functional MRI (fMRI), electroencephalography (EEG), and magnetoencephalography (MEG) on humans have suggested that, apart from the temporal cortical regions, the medial prefrontal cortex (MPF) is also involved in TP [17-19]. The fMRI studies by Meltzer and colleagues [17,18] demonstrated deactivation in the temporal cortex and MPF as well as the hippocampus, and activation in the parietal cortex during TP. In addition, their EEG analysis also reported increased theta power in MPF and decreased alpha power in the precuneus during TP. They suggested that the change in theta power could be associated with memory or cognitive demands. Mills and colleagues [19] revealed robust activations in the right hippocampus and frontal cortex during TP. These data have suggested that the prefrontal cortex, especially $\mathrm{MPF}$ is involved in TP.

To date, the roles of the prefrontal cortical regions in TP are still unclear. To address this issue, we examined the response characteristics of prefrontal neurons while a monkey performed a TP task. Because the activations of both fMRI and MEG studies were rather robust, we surveyed a relatively wide area in the prefrontal cortex and compared the response properties between the subregions. In addition, to ascertain whether the 
responses of the prefrontal neurons would play a specific role in TP, we also examined the responsiveness of the prefrontal neurons during a visual delayed matching-to-sample (DMS) task and compared the responsiveness between the TP and DMS tasks. 


\section{Material and methods}

\subsection{Subjects and apparatus}

Two adult male macaque monkeys (monkey C, Macaca fuscata, $7 \mathrm{~kg}$; monkey M, Macaca mulatta, $10 \mathrm{~kg}$ ) were used in this study. Water was withheld before each daily session, and was given as a reward in an experimental room. Supplemental water and vegetables were given after the session. Experiments were conducted according to the Guide for the Care and Use of Laboratory Primates of the Primate Research Institute, Kyoto University (2002, 2010) and Guide for Care and Use of Laboratory Animals of the National Research Council (1996). This study was approved by the ethics committee of PRI, Kyoto University.

\subsection{Behavioral tasks}

Each monkey was seated on a primate chair in a dark soundproof room facing a 17-inch cathode ray tube (CRT) monitor (FlexScan T565, Nanao, Ishikawa) placed at a distance of $30 \mathrm{~cm}$. The monkey's head was fixed painlessly to the primate chair with a head-restraining device. The behavioral tasks were controlled by a personal computer (PC-9821Xe10, NEC, Tokyo).

The monkeys were trained on a TP task (Fig. 1a left). In the TP task, a trial started with the presentation of a white fixation spot $\left(0.3^{\circ} \times 0.3^{\circ}\right)$ at the center of the CRT monitor. If the monkey fixated it for 2500-3000 ms, choice cues (target and nontarget stimuli, $2^{\circ} \times 2^{\circ}$ ) were presented on the left and right sides of the fixation spot (eccentricity: $9^{\circ}$ ). When the monkey continued to fixate the fixation spot for another $500-700 \mathrm{~ms}$, the spot disappeared. If the monkey made a saccade to the target stimulus within $1000 \mathrm{~ms}$ and fixated the target for $200 \mathrm{~ms}$, the PC emitted a beep sound for $200 \mathrm{~ms}$ and a drop of water was delivered after $100 \mathrm{~ms}$ of prereward period. The beep sound informed the monkey whether the response was correct or not. When the monkey made an erroneous saccade to the nontarget stimulus, the visual stimuli disappeared and the monitor turned black. Thus, the monkey could recognize whether the current saccade was correct or not just after the saccadic response. If the monkey had broken the fixation before the end of the trial, a blank screen was immediately presented for $2000 \mathrm{~ms}$. The next trial started after an intertrial interval of $2000 \mathrm{~ms}$. Trials with a fixation break before the offset of the fixation spot were regarded as 
incomplete trials, and the data were not analyzed in detail in the present study.

The monkey's eye position was measured by an infrared camera (R-21C-AB, RMS, Hirosaki) at a sampling rate of $250 \mathrm{~Hz}$. When the monkey's eye position was in a fixation window, a square of $4^{\circ} \times 4^{\circ}$ centering on the fixation spot, we regarded that the monkey fixated the fixation spot. If the monkey's eye position was out of the window, we considered that the monkey failed to fixate the spot. We set a target window, a square of $5^{\circ} \times 5^{\circ}$ on each side centering on the target stimulus. If the monkey's eye position was in the target window, we regarded that the monkey made a saccadic eye movement to the target.

In the TP task, the monkey was required to choose the target stimulus on the basis of a predefined rule. We used 3 visual stimuli for each monkey (Fig. 1b). For a set of stimuli A, B, and C, the rule for choice was defined as $\mathrm{A}+/ \mathrm{B}-, \mathrm{B}+/ \mathrm{C}-$, and $\mathrm{C}+/ \mathrm{A}-$, where the "+" and "-" indicate the target and the nontarget, respectively (Fig. 1c). This rule was fixed throughout the entire period of the experiments. Consequently, there were 6 trial conditions; 3 stimulus pairs $(\mathrm{A}-\mathrm{B}, \mathrm{B}-\mathrm{C}$, or $\mathrm{C}-\mathrm{A}) \times 1$ predetermined target stimulus $\times 2$ target locations (left or right).

The monkey was trained on a DMS task as a control task (Fig. 1a right). The same 3 visual stimuli as those in the TP task were used for each monkey. In the DMS task, a white fixation spot was presented at the center of the monitor. If the monkey fixated it for $1000 \mathrm{~ms}$, a visual sample stimulus $\left(2^{\circ} \times 2^{\circ}\right)$ was presented at the center for $500 \mathrm{~ms}$. After a delay period of $1000-$ $1500 \mathrm{~ms}$, choice cues were presented. In the DMS task, the monkey had to choose the sample stimulus as the target stimulus.

Depending on the sample, either A or B could be the target for the $\mathrm{AB}$ pair in the DMS task. Thus, there were 12 trial conditions in total; 3 stimulus pairs $(\mathrm{A}-\mathrm{B}, \mathrm{B}-\mathrm{C}$, or $\mathrm{C}-\mathrm{A}) \times 2$ target stimuli $\times 2$ target positions (left or right). In half of the DMS trials, the target was the same as in the TP task (designated as "congruent" trials), whereas in the other half of the DMS trials, the target assignment was the opposite (designated as "incongruent" trials) (Fig. 1c). In the congruent trials, after the onset of the cue presentation, the same visual stimuli were presented, the same target stimulus was chosen, and the saccadic eye movement to the same direction occurred as in the TP task. The congruent and incongruent trials were mixed 
pseudorandomly.

For both tasks, in each trial, a pair of stimuli was selected pseudorandomly from the set of 3 stimuli. The location of the target stimulus (left or right) was also assigned pseudorandomly. The 2 tasks were interleaved in a blockwise manner. A block was completed when the monkey achieved at least 10 correct trials for the each trial condition (60 trials in a block for the TP task and 120 trials in a block for the DMS task, at the minimum). Details of the task training are explained in Supplementary data 1.

\subsection{Recording procedure}

Action potentials of single neurons were recorded extracellularly using glass-coated Elgiloy microelectrodes (0.5-2 $\mathrm{M} \Omega$ ) and amplified by an amplifier (MEG-6108, Nihon Kohden, Tokyo). The electrodes were inserted using a grid system. Action potentials were isolated and converted to pulses using a spike waveform detector (Multispike Detector, Alpha Omega Engineering, Nazareth). The data of action potentials and task events were stored in the hard disk of the PC. We examined the responsiveness of the neurons tested with $\geq 10$ correct trials in the TP task; the data for the neurons tested with $<10$ correct trials were discarded from further analysis.

The positions of the recording sites and angle of the recording chamber were determined on the basis of MR images. In addition, the location of the frontal eye field was determined by intracortical microstimulations (ICMS, 0.8-ms pulse duration, 3.2-ms pulse interval, 50 pulses/train, $80 \mu \mathrm{A}$ ) out of behavioral tasks [20,21]. After all recordings were completed, to confirm the recording sites, several electrolytic lesions were made in the prefrontal cortex by passing an anodal current (30 s, $20 \mu \mathrm{A})$ (Figs. 2a and 2b).

\subsection{Data analysis}

We analyzed the firing rates in 4 task periods. The control period was a $300-\mathrm{ms}$ period from $500 \mathrm{~ms}$ after the fixation onset. The initial $500 \mathrm{~ms}$ was discarded to avoid the effects of saccadic eye movements to the fixation spot. The cue period was a 300-ms period from $100 \mathrm{~ms}$ after the presentation of the choice cues. The initial $100 \mathrm{~ms}$ was excluded to compensate for the latency of typical visual responses in PF [22]. The presaccadic period was a 
300-ms period immediately before the saccade onset. The postsaccadic period was a $300-\mathrm{ms}$ period immediately after the saccade offset. The saccade onset was defined as the time when the monkey's eye position went out of the fixation window, and the saccade offset was defined as the time when the monkey's eye position fell within the target window.

Because the monkey was expected to perceive the visual choice cue, choose a target on the basis of the task rule, and make a saccade to the target during the cue and presaccadic periods, we first compared the neuronal firing during these periods with that during the control period. If the firing during the cue or presaccadic period was significantly different from that during the control period ( $\alpha=0.01$, Steel test), the neuron was regarded as task responsive. Then, to evaluate the response selectivity of the responsive neurons, we analyzed the neuronal firing in each period with a 2-way analysis of variance (ANOVA) with Tukey's honestly significant difference (HSD) post hoc test $(\alpha=0.05)$. The factors were location (left or right) and shape (A, B, or C) of the target stimulus. If the effect of any factor or interaction was significant, we considered that the neuron exhibited response selectivity.

To examine whether the neuronal activity was TP-task dependent or not, we compared the firing rate of each neuron during the TP task with that during the DMS task. In this analysis, we used the data from the congruent trials because the physical stimulations were the same and the direction of the saccade response was the same. The only difference was the rule to solve the task. We analyzed the neuronal firing, in each period, of both tasks using a 3-way ANOVA with Tukey's HSD post hoc test $(\alpha=0.05)$. The factors were task type (TP or DMS), location (left or right), and shape (A, B, or C). We analyzed the data from not only the "task responsive" neurons but also "task unresponsive" neurons because some neurons showed significantly differential firing between the tasks even during the control period (a 300-ms period from $500 \mathrm{~ms}$ after the fixation onset before visual stimulation).

We also found that some neurons changed their activity after saccadic eye movement to the target stimulus in the TP task. We analyzed the neuronal firing in the postsaccadic period of both tasks, using a 3 -way ANOVA with Tukey's HSD post hoc test $(\alpha=0.05)$, as we had done for the cue and presaccadic periods. Then, we examined how these neurons behaved when the monkey committed an erroneous saccade; i.e., in an error trial. 
Some neurons changed their firing rate during the control period, depending on the task type. We compared the neuronal firing during the control period between the TP and DMS tasks, using Wilcoxon rank sum test $(\alpha=0.05)$.

Spike density functions were computed by convolution of the spike trains with a Gaussian kernel $(\sigma=30 \mathrm{~ms})$, using a resolution of $1 \mathrm{~ms}$.

Success rate during the recording of each neuron was calculated separately for the TP task, and the congruent trials and the incongruent trials of the DMS task.

\subsection{Histological procedures}

The recording sites were histologically determined on a series of coronal sections. After the completion of all the recording sessions, the monkey was deeply anesthetized with ketamine [2.5 $\mathrm{mg} / \mathrm{kg}$, intramuscular (i.m.)], medetomidine (0.1 mg/kg, i.m.), and pentobarbital sodium (30 mg/kg, intravenous). The brain was perfused transcardially with $0.1-\mathrm{M}$ phosphate buffered saline, and then with $0.1-\mathrm{M}$ phosphate buffer containing $8 \%$ formalin. Prior to formalin perfusion, 3 stainless steel needles were inserted at known coordinates of the recording chamber (Figs. 2a and 2c). The brain was postfixed in the same fresh fixative for 1 day, saturated with $30 \%$ sucrose at $4^{\circ} \mathrm{C}$, and then cut serially into coronal sections $100-\mu \mathrm{m}$-thick on a freezing microtome. The sections were Nissl-stained with 1\% Neutral Red. Locations of the recording sites in this monkey were determined using the marks of the electrolytic lesions and the tracks of the needles on the stained sections as references.

\section{Results}

\subsection{Behavioral data}

The 2 monkeys often failed to fixate the fixation spot during a trial. The mean percentage of the incomplete trials with a fixation break before the disappearance of the fixation spot in the TP task and in the congruent and incongruent trials of the DMS tasks were 20.9\%, 32.9\%, and $32.3 \%$ for monkey C, and 23.4\%, 31.5\%, and 29.4\% for monkey M, respectively. As for the TP task, the rates of incomplete trials for the three stimulus pairs (AB, $\mathrm{BC}$, and CA) were $21.0 \% \pm 6.6 \%, 17.5 \% \pm 5.1 \%$, and $20.7 \% \pm 5.6 \%$ for monkey $\mathrm{C}$, and $23.2 \% \pm 6.7 \%, 24.2 \pm 5.2 \%$, and $21.4 \% \pm 4.1 \%$ for monkey $\mathrm{M}$, 
respectively. The rates were not significantly different among stimulus pairs in monkey $\mathrm{M}\left(\chi^{2}=1.92, P>0.05\right.$, Kruskal-Wallis test). For monkey $\mathrm{C}$, there was a marginally significant difference among stimulus pairs $\left(\chi^{2}=6.57, P=\right.$ 0.037). These incomplete trials with a fixation break were excluded from further analysis.

Otherwise, monkeys were expertized in both TP and DMS tasks prior to the beginning of the recording experiments, and made few erroneous choices. The rates of correct responses (median \pm quartile deviation) for the TP task, and the congruent and incongruent trials of the DMS tasks, were $96.0 \% \pm 1.5 \%, 98.1 \% \pm 1.0 \%$, and $94.6 \% \pm 2.4 \%$ for monkey $\mathrm{C}$, and $90.3 \% \pm$ $3.1 \%, 98.8 \% \pm 1.0 \%$, and $91.8 \% \pm 2.5 \%$ for monkey $\mathrm{M}$, respectively. The rate of correct responses of the congruent trials of the DMS task was significantly higher than those of the TP task and the incongruent trials of the DMS task in both monkeys (Monkey C, $\chi^{2}=63.9, P<0.001$; Monkey M, $\chi^{2}=116.27, P<$ 0.001, Kruskal-Wallis test with Tukey's HSD post hoc test). In the TP task, the rates of correct responses for the three stimulus pairs ( $\mathrm{AB}, \mathrm{BC}$, and $\mathrm{CA})$ were $96.9 \% \pm 2.3 \%, 95.7 \% \pm 2.4 \%$, and $97.1 \% \pm 2.6 \%$ for monkey $\mathrm{C}$, and $91.2 \%$ $\pm 5.5 \%, 91.3 \% \pm 4.5 \%$, and $91.9 \% \pm 4.8 \%$ for monkey $\mathrm{M}$, respectively. These rates were not significantly different among stimulus pairs in both monkeys (Monkey C, $\chi^{2}=3.93, P>0.05$; Monkey M, $\chi^{2}=0.76, P>0.05$, KruskalWallis test).

\subsection{Neuronal data}

A total of 221 neurons were examined in the TP task (151 and 70 in monkeys $\mathrm{C}$ and $\mathrm{M}$, respectively). On the basis of histological examination, the recording sites were divided into 3 prefrontal regions: PS (both the dorsal and ventral banks and its lips); DC (the convexity dorsal to PS); and MPF (the medial wall above the cingulate sulcus, which extended somewhat to the dorsal bank of the cingulate sulcus) (Fig. 2d). Among the 221 neurons, 72, 90, and 59 were located in PS, DC, and MPF, respectively. Out of the 221 neurons, 190 were also tested with the DMS task (120 and 70 in monkeys C and $\mathrm{M}$, respectively). Among them, 64, 72, and 54 neurons were located in PS, $\mathrm{DC}$, and MPF, respectively.

\subsubsection{Neuronal responsiveness in the TP task}

3.2.1.1. Response profiles. Many prefrontal neurons showed changes in 
activity during the cue and/or presaccadic periods of the TP task. Examples of each type of response are shown in Fig. 3. The first type of neuron responded during the cue period. An MPF neuron (Fig. 3a) showed a clear response just after the cue was presented. This response soon ceased in the cue period. We designated neurons of this type as cue-type (C-type) neurons. The second type of neuron changed its firing rate during the presaccadic period. A PS neuron (Fig. 3b) gradually increased its firing rate and reached a peak during the presaccadic period. We designated neurons of this type as presaccade-type (P-type) neurons. The third type of neuron started to respond in the cue period and continued the response during the presaccadic period. A PS neuron (Fig. 3c) increased its firing rate just after the cue onset and the response continued until the monkey made a saccade. We designated neurons of this type as cue-and-presaccade-type (CP-type) neurons.

Among the 72 PS, 90 DC, and 59 MPF neurons tested, 34 (47\%), 29 (32\%), and $40(68 \%)$ neurons were responsive during the cue or presaccadic period. The ratio of the responsive neurons to the tested neurons was significantly different among the 3 regions $\left(\chi^{2}=18.14, P<0.001, \chi^{2}\right.$ test); more in MPF and less in DC (MPF, $P<0.001$; DC, $P<0.001$, residual analysis as the post hoc test). The ratios of $\mathrm{C}$-type, $\mathrm{P}$-type, and $\mathrm{CP}$-type neurons to the tested neurons were 9 (13\%), $11(15 \%)$, and $10(14 \%)$ in PS neurons, $7(8 \%), 12(13 \%)$, and 9 (10\%) in DC neurons, and $10(17 \%), 14$ (23\%), and $16(27 \%)$ in MPF neurons, respectively (Figs. S1 and S2). The ratios of each type of neuron were examined. The ratios of $\mathrm{C}$-type and $\mathrm{P}$-type neurons were not different among the 3 prefrontal regions (C-type, $\chi^{2}=2.94$, $P>0.05$; P-type, $\left.\chi^{2}=2.92, P>0.05\right)$, whereas the ratio of $\mathrm{CP}$-type neurons was significantly different among the 3 regions $\left(\chi^{2}=8.14, P=0.016\right)$; more in MPF and less in DC (MPF, $P=0.006$; DC, $P=0.049)$. Four PS neurons and 1 DC neuron responded during the cue period under some trial conditions, and during the presaccadic period under other trial conditions. Thus, we could not classify these 5 neurons.

During the TP task, there were more responsive neurons, especially CP-type neurons, in MPF than in the other regions.

3.2.1.2. Selectivity for target location and shape. Fig. 3a shows a C-type neuron, which exhibited a strong response to the shape $\mathrm{B}$ as a target during the cue period, but only weak responses to the shapes $\mathrm{A}$ and $\mathrm{C}(F(2,78)=$ 
5.58, $P=0.005,2$-way ANOVA; right panel). The CP-type neuron shown in Fig. 3c also showed a shape-dependent responses. This neuron increased its firing rate in response to shapes $\mathrm{A}$ and $\mathrm{C}$ as a target during the cue period, but the increase was less clear in response to shape $\mathrm{B}(F(2,60)=6.29, P=$ 0.003; right panel). The P-type neuron shown in Fig. $3 \mathrm{~b}$ increased its firing rate before the monkey made a saccade to the right target but not to the left target $(F(1,54)=15.24, P<0.001$; right panel $)$.

To examine the selectivity of the responses for shape and location, we applied a 2-way ANOVA, where the factors were shape (A, B, or C) and location (left or right), for each neuronal response during the cue or presaccadic period, separately.

Among the neurons showing responses during the cue period (C-type and CP-type neurons), a few exhibited shape-dependent response: 4 PS neurons (6\%), 1 DC neurons (1\%), and $1 \mathrm{MPF}$ neurons (2\%). This was also the case for location-dependent response: 3 PS (4\%), 3 DC (3\%), and $4 \mathrm{MPF}$ (7\%) neurons. In addition, 3 PS (4\%), 2 DC (2\%), and 2 MPF (3\%) neurons showed a significant interaction between shape and location.

Among the neurons responsive during the presaccadic period (P-type and CP-type neurons), again only a few neurons exhibited shape-dependent response: 3 PS (4\%), 1 DC (1\%), and 2 MPF (3\%) neurons. However, approximately $10 \%$ of the neurons showed location-dependent responses; 8 PS (11\%), 5 DC (6\%), and 7 MPF (12\%) neurons. Three PS (4\%) and $4 \mathrm{MPF}$ (7\%) but no DC neurons exhibited a significant interaction between shape and location.

Some prefrontal neurons changed their firing rate depending on the shape and/or location of the target.

\subsubsection{Task dependency.}

To examine whether the recorded neurons played some unique role in the TP task, we tested 190 neurons in the DMS task as well as in the TP task, and compared the responsiveness. As described in 2.2. Behavioral tasks, we used the same visual stimuli in both tasks, and required the monkeys to make a saccade to the same target in the TP task and in the "congruent" trials of the DMS task, so that we could directly compare the responsiveness between the TP and DMS tasks (Fig. 1c). 
3.2.2.1 Task-dependent responses. As shown in Fig. 4a, a C-type neuron in PS showed shape-independent (left) and location-independent (middle) responses during the cue period (shape, $F(2,228)=0.35, P>0.05$; location, $F$ $(1,228)=0.68, P>0.05,3$-way ANOVA). However, the response was significantly stronger during the TP task than during the DMS task ( $F(1$, $228)=73.97, P<0.001$; right). Fig. $4 \mathrm{~b}$ shows the responses of a CP-type neuron in MPF. This neuron exhibited shape-independent (left) but location-dependent (middle) responses during the cue period, and the firing continued to the onset of the saccadic eye movements (shape, $F(2,300)=$ 1.17, $P>0.05$; location, $F(1,300)=11.68, P<0.001)$. Its firing was stronger in the TP task than in the DMS task $(F(1,300)=80.23, P<0.001$; right $)$. Fig. $4 \mathrm{c}$ shows the responses of a P-type neuron in PS, showing shape-independent (left) but location-dependent (middle) responses during the presaccadic period (shape, $F(2,108)=1.31, P>0.05$; location, $F(1,108)=6.61, P=$ 0.012). This neuron fired more strongly in the TP task than in the DMS task $(F(1,108)=5.70, P=0.019$; right $)$. On the other hand, a P-type PS neuron, shown in Fig. 4d, exhibited shape-dependent (left) and location-dependent (middle) responses during the presaccadic period, while the neuron showed a task-independent response (shape, $F(2,276)=10.44, P<0.001$; location, $F$ $(1,276)=0.55, P>0.05$; task, $F(1,276)=0.01, P>0.05$; right $)$.

To examine the task dependency of the responses, we applied a 3 -way ANOVA, where the factors were task (TP or DMS), shape (A, B, or C), and location (left or right), for each neuronal response during the cue or presaccadic period. Results of the ANOVA are summarized in Table 1.

If a neuron changed its firing rate depending on the task type, that is, the main effect of task on its firing was significant, we designated the neuron as a "task-dependent" neuron. During the cue period, the number of the task-dependent neurons in MPF was significantly greater than those in DC and in PS ( $\chi^{2}=8.65, P=0.012$, $\chi^{2}$ test; MPF, $P=0.004$, residual analysis). During the presaccadic period, the number was not significantly different among the 3 regions $\left(\chi^{2}=0.99, P>0.05\right)$. Then we compared the response magnitudes of these task-dependent neurons between the 2 tasks. As shown in Table 2, the number of neurons more active in the TP task was not significantly different from that of neurons more active in the DMS task $(P>$ 0.05, Binomial test). During the presaccadic period in PS, however, more neurons responded more strongly in the TP task $(P=0.004)$. 
Many prefrontal neurons exhibited task-dependent changes in activity, and there were more task-dependent neurons in MPF than in the other 2 regions.

3.2.2.2. Shape-dependent and location-dependent responses. As already described, like neurons shown in Figs. 4b, 4c, and 4d, some prefrontal neurons exhibited target shape-dependent and/or location-dependent responses. The numbers of neurons exhibiting shape-dependent and location-dependent responses are summarized in Table 1. The ratios of shape-dependent neurons were not significantly different among the 3 regions (cue period, $\chi^{2}=3.71, P>0.05$; presaccadic period, $\chi^{2}=4.51, P>0.05$, $\chi^{2}$ test). By contrast, the ratios of the location-dependent neurons were significantly different among the 3 regions during the presaccadic period, but not during the cue period (cue period, $\chi^{2}=1.64, P>0.05$; presaccadic period, $\left.\chi^{2}=11.69, P=0.003\right)$. There were more location-dependent neurons in PS and MPF, and less in DC (PS, $P=0.028$; MPF, $P>0.05 ; \mathrm{DC}, P<0.001$, residual analysis).

Prefrontal neurons in the 3 regions became more selective for target location during the presaccadic period than during the cue period. The numbers of neurons exhibiting location-dependent responses in all the 3 prefrontal regions were larger during the presaccadic period than that during the cue period $\left(\chi^{2}=21.43, P<0.001\right.$, McNemar's test). Among the 3 regions, in $\mathrm{PS}$ and $\mathrm{MPF}$ but not in $\mathrm{DC}$, the numbers were significantly greater during the presaccadic period than during the cue period (PS, $\chi^{2}=$ 13.24, $P<0.001$; DC, $\left.\chi^{2}=1.6, P>0.05 ; \mathrm{MPF}, \chi^{2}=8.07, P=0.005\right)$. The number of neurons exhibiting shape-dependent responses was not different between the cue and presaccadic periods $(P>0.05)$.

Among the location-dependent neurons, some were task-dependent (Figs. 4b and 4c), but others were not (Fig. 4d). During the cue period, the number of location-dependent neurons was small in total, so we did not further analyze the task dependency of these neurons. During the presaccadic period, the location-dependent but task-independent neurons were more frequently observed in PS (14, 22\%), compared with DC (5, 7\%) and $\mathrm{MPF}(7,13 \%)\left(\chi^{2}=8.02, P=0.029, \chi^{2}\right.$ test; PS, $P=0.005$, residual analysis). By contrast, the number of the location-dependent and task-dependent neurons was larger in MPF (10, 19\%) than in DC (3, 4\%) and 
PS $(8,13 \%)$ during the presaccadic periods $\left(\chi^{2}=6.67, P=0.035 ; \mathrm{MPF}, P=\right.$ $0.039)$.

Prefrontal neurons showed responses selective for target shape and location during the tasks. They became more selective for target location during the presaccadic period, especially in PS and MPF. During the presaccadic period, neurons selective for the target location were more task-independent in PS, whereas they were more task-dependent in MPF.

3.2.2.3. Task interaction neurons. Some prefrontal neurons changed their response profile between the TP and DMS tasks. Examples of the responses of such a neuron are shown in Fig. 5. The neuron shown in Fig. 5a is the same neuron shown in Fig. 3a. This C-type neuron showed shape-dependent responses. In the TP task, it responded strongly to the shape $\mathrm{B}$, but weakly to the shapes A and C. By contrast, the same neuron responded only weakly to the shapes A, B, and C in the DMS task. The selectivity for target shape was observed only in the TP task $(F(2,156)=3.47, P=0.033$, 3-way ANOVA). The neuron shown in Fig. 5b gradually increased its firing rate toward the saccade onset in both tasks. This CP-type neuron showed location-dependent responses in the TP task. It showed stronger responses under the conditions when the monkey made saccadic eye movements to shape $\mathrm{B}$ on the right than to the same target on the left. By contrast, in the DMS task, the neuronal responses were not different among conditions $(F(2,204)=4.80, P=0.009)$.

Like these neurons, some prefrontal neurons exhibited response selectivity for shape and/or location only in 1 task (TP or DMS), or changed the selective profile depending on the task type. For these neurons, the results of the ANOVA revealed that the interaction of (task $\times$ shape), (task $\times$ location), or (task $\times$ shape $\times$ location) was significant. Therefore, we designated these neurons as "task interaction" neurons, for convenience.

The numbers of task interaction neurons during the cue period were $13(20 \%), 9(13 \%)$, and $10(19 \%)$ in PS, DC, and MPF, respectively. Those during the presaccadic period were $9(14 \%), 13(18 \%)$, and $16(30 \%)$ in PS, DC, and MPF, respectively.

These neurons apparently conveyed information about the shape and/or location of the target specifically for a certain task.

\subsubsection{Postsaccadic response}


The postsaccadic period was the $300-\mathrm{ms}$ period from the offset of the saccade. Some prefrontal neurons responded during this period, and so we analyzed their response characteristics. Note that the monkey was able to become aware of whether the current response was correct or not just after the saccade (see Materials and Methods). The PS neuron shown in Fig. 6a was more active in the TP task than in the DMS task in the postsaccadic period $(F(1,228)=75.91, P<0.001,3$-way ANOVA $)$ Fig. $6 \mathrm{~b}$ is an example of a location-dependent neuron recorded in DC, which was more activated after a rightward saccade $(F(1,156)=118.93, P<0.001)$. The number of neurons showing task-dependent, location-dependent, and shape-dependent postsaccadic response was 23 (36\%), 20 (31\%), and 7 (11\%) in PS; 24 (33\%), $25(35 \%)$, and $6(8 \%)$ in DC; and $22(41 \%), 21(39 \%)$, and $4(7 \%)$ in MPF; respectively. In addition, 13 (20\%) PS, 20 (28\%) DC, and 6 (11\%) MPF neurons showed task-interaction.

To further study the characteristics of the postsaccadic responses, we examined the responses of these neurons in erroneous trials. Generally, however, the performance of the monkey was quite good (over $90 \%$ for the TP task); therefore there were very few erroneous trial. Thus, we analyzed a change in activity for only 10 neurons. Two examples of the responses in erroneous trials are shown in Fig. 7.

The neuron shown in Fig. 7a fired strongly after rightward saccades, but not after leftward saccades, in correct trials $(F(1,156)=27.97, P<0.001$, 3-way ANOVA; left panel). In erroneous trials, the neuronal firing was observed again after erroneous rightward saccades, but not leftward saccades (right panel), although we could not conduct any statistical test because of the small number of trials. We confirmed similar response patterns in 8 out of 10 neurons. In the remaining 2 neurons, response characteristics changed drastically. The neuron shown in Fig. 7b responded just after the saccadic eye movements in correct trials and the firing rate was higher in the rightward saccade trials than in the leftward trials $(F(1,192)=$ 7.20, $P=0.008$; left panel). In erroneous trials, the same neuron fired vigorously at almost the same timing (right panel). Another neuron responded only after the leftward saccades in correct trials, but the same neuron ceased to fire completely after incorrect saccades.

On the basis of these data, some of the postsaccadic responses reflected the task type, the direction of the saccade, the shape of the target, 
and the outcome of the current trial.

\subsubsection{Task-dependent activity starting before the cue presentation}

Even in the control period, a group of neurons showed a differential level of activity between the 2 tasks (Fig. 8). The numbers of such neurons were 12 (19\%) in PS, 19 (26\%) in DC, and 21 (39\%) in MPF. The number of these neurons in MPF was significantly greater than those in DC and PS $\left(\chi^{2}=6.03\right.$, $P=0.049, \chi^{2}$ test; MPF, $P=0.025$, residual analysis). Soon after the monkey fixated the fixation spot in the DMS task, the DC neuron shown in Fig. 8a exhibited higher activity $(Z=3.12, P=0.002$, Wilcoxon rank sum test), which continued to the cue periods. Among the neurons showing task-dependent activity in the control period, over half continued to be task-dependent, at least until the cue period; 6 (9\%) in PS, 12 (17\%) in DC, and $13(24 \%)$ in MPF. We designated these neurons as "task state" neurons. Like the DC neuron shown in Fig. 8b, some of the task state neurons continued to show task-dependent firing throughout a trial, including the cue, presaccadic, and postsaccadic periods. Just after the monkey fixated the fixation spot, before the control period, the DC neuron already showed a higher level of firing in the TP task than in the DMS task $(Z=-5.16, P<0.001$; left $)$, and the differential firing continued throughout a trial after the monkey made a saccadic eye movement (middle and right). The numbers of neurons showing task-dependent activity throughout a trial were 4 (6\%) in PS, 7 (10\%) in DC, and $7(13 \%)$ in MPF. 


\section{Discussion}

In the present study, we found that many prefrontal neurons responded during the execution of TP. We examined response characteristics of neurons in a relatively wide portion of the monkey prefrontal cortex, i.e., PS, DC, and MPF. In all these regions, at least one-third of the neurons examined showed a change in activity during the cue or presaccadic period of the TP task. These data suggest that some prefrontal neurons function in the execution of the TP task. Among these regions, there were more TP task-responsive neurons in MPF than in PS and DC. This suggests that the MPF would make more contribution to the execution of the TP task, compared to the DC or PS. Consistent with our present data, recent brain imaging studies have reported the involvement of MPF during TP [17-19].

We also examined the neuronal responses in the DMS task, where the same visual stimuli as in the TP task were used. We compared the neuronal responses between the TP task and the congruent trials of the DMS task because, in these 2 conditions, the monkey made saccades to the same target for each pair of choice cues. Among the neurons tested, some neurons showed differential response between the 2 tasks (the task-dependent neurons). Almost all of these neurons responded similarly in the congruent and incongruent trials of the DMS task (Supplementary data 2). These neurons would convey information specific to each task. In addition, these task-dependent neurons were more abundant in MPF than in the other regions. Among the task-dependent neurons in the MPF, nearly equal number of neurons was activated by the TP and DMS tasks (15 and 13 neurons, respectively). These data suggest that MPF is important for applying the appropriate rule, either the TP or DMS.

\subsection{Target selection}

Many prefrontal neurons responded during the cue period when the monkey perceived the visual cue (target and nontarget stimuli), and selected a target on the basis of the rule of the current task. Some prefrontal neurons exhibited target shape-dependent and/or target location-dependent responses. Many studies reported neuronal activities in PF that were selective for the stimulus features before the implementation of behavior [23-25]. In the present study, some shape-dependent or location-dependent neurons showed task-dependent profiles; i.e., these neurons exhibited 
response selectivity for shape and/or location in only 1 task (TP or DMS), or changed the selective profile depending on the task type. These neurons apparently conveyed information about the location and/or shape of the target in a context-dependent manner.

On the other hand, some neurons (task state neurons) started to show a differential level of activity between the TP and DMS tasks before the presentation of the choice cue, or the sample in the DMS task, and kept the differential activity at least until the cue period. Moreover, some of these neurons continued to show differential firing throughout a trial. The signals from these "task state" neurons could differentiate the 2 tasks and inform the current ongoing task state. The signals could be used for the generation of the task-dependent response profiles of the "task-dependent" and "task interaction" neurons. Interestingly, the number of the neurons showing task dependency in the control period (that is, the fixation period) was significantly greater in MPF than in PS and DC. Approximately one-third of the task-dependent neurons in the control period consecutively showed task-dependent activity in each region throughout a trial.

Our present data suggest that MPF is more important for the selection of the target, on the basis of the rule of the current task, than are PS and DC. In line with our current results, a previous study suggested the possible involvement of MPF in target-selection based on context and external stimuli [26].

\subsection{Response execution}

Many prefrontal neurons also responded during the presaccadic period when the monkey prepared the saccade response to the target after selecting it. Many neurons showed responses selective for the target locations. Compared with the cue period, the prefrontal neurons became more selective for the target location during the presaccadic period. Among the 3 prefrontal regions, there were more location-dependent neurons in PS and MPF than in DC during the presaccadic period, but not during the cue period. The signals from these location-dependent neurons during the presaccadic period could be used for the generation or preparation of the saccade response to the target. The ratios of the location-dependent and task-dependent neurons in MPF were higher than in the other 2 regions. On the other hand, the ratios 
of the location-dependent and task-independent neurons in PS were higher than in the other 2 regions. These results might suggest that the roles of these location-dependent neurons differ between MPF and PS. MPF could be more related to the context-dependent representation of the target. In contrast, PS could be more related to the context-independent representation of the target. As regards DC, relatively few task-dependent, location-dependent, and shape-dependent neurons were found in this region compared with the other 2 regions; therefore DC's role in target selection and response execution is not clear.

In the present study, MPF contained more CP-type neurons than the other regions. These neurons would presumably play important roles in multiple aspects of task execution, from target selection to response execution.

\subsection{Feedback and the optimization of behavior}

In the postsaccadic period, a certain number of neurons in each of the 3 regions were task-dependent, location-dependent, or shape-dependent. Analysis of the erroneous trials revealed that most of the location-dependent neurons changed responses depending on the saccade direction, but not the location of the correct target. These postsaccadic responses would represent different aspects of the decision that had been made (to follow a specific rule, to choose a specific target, to make a saccade to a specific location), at the time when the outcome was announced. Interestingly, we also found neurons that changed their response drastically between correct (rewarded) and erroneous (unrewarded) trials in this period. Altogether, these postsaccadic responses would represent any aspects of the previous decision, regardless of the relevance in the present task context, and its outcome. Such responses would be useful in reinforcing or extinguishing the rewarded or unrewarded decisions. Similar "post-response" activities in the lateral prefrontal cortex have been reported previously [27,28].

4.4. Possible interactions between PF and medial temporal lobe (MTL) in the TP task

Previous studies in human patients and nonhuman primates have indicated that MTL, including the hippocampus, is crucial for learning of TP $[11,29,30]$. On the other hand, functional imaging studies in human subjects suggested 
that MPF is also important in the performance of the TP task after sufficient practice $[17,18]$.

In the present study, we analyzed the single neuronal activities in the monkey PF after the monkeys had fully mastered the TP rule. The results suggested the involvement of $\mathrm{PF}$, especially $\mathrm{MPF}$, in the TP task performance at the postlearning stage. Previous imaging studies reported that the signal from MTL was also changed during the performance of the TP task [17-19]. These results suggest that PF, especially MPF, together with MTL, functions in the performance of the TP task.

Anatomically, MPF in this study roughly corresponds to the medial subdivision of area 9 extending to the dorsal bank of the cingulate sulcus [31]. This area would have disynaptic bidirectional connections with MTL (entorhinal and perirhinal cortices) via the anterior cingulate gyrus, including areas 24, 32, and 25 [32-35]. Therefore, MPF may possibly exert top-down control to MTL, retrieving mnemonic information through these neuronal pathways for behavioral control [36-39].

\section{Conclusion}

The present study showed that many neurons in PF, especially those in MPF, responded during the performance of the TP task. For target selection based on the rule and visual information, MPF would play the most important role. Then, PS, together with MPF, would participate in the response preparation. The results of this study, together with those from previous studies, suggest that PF, especially MPF, work together with MTL in the execution of the TP task.

\section{Acknowledgements}

This work was supported by Grants-in-Aid for Scientific Research on Priority Areas (20019021 to S.M.), and by Global COE program A06 (to M.N.) from the Ministry of Education, Culture, Sports, Science and Technology of Japan. The authors would like to thank Enago (www.enago.jp) for the English language review. 


\section{References}

[1] Spence KW. The nature of the response in discrimination learning. Psychol Rev 1952;59(1):89-93.

[2] Rudy JW. Elemental and configural associations, the hippocampus and development. Dev Psychobiol 1991;24(4):221-236.

[3] Rudy JW, Sutherland RJ. Configural and Elemental Associations and the Memory Coherence Problem. J Cogn Neurosci 1992;4(3):208-216.

[4] Rudy JW, Keith JR, Georgen K. The effect of age on children's learning of problems that require a configural association solution. Dev Psychobiol 1993;26(3):171-184.

[5] Rudy JW, Sutherland RJ. Configural association theory and the hippocampal formation: An appraisal and reconfiguration. Hippocampus 1995;5(5):375-389.

[6] O'Reilly RC, Rudy JW. Conjunctive representations in learning and memory: Principles of cortical and hippocampal function. Psychol Rev 2001;108(2):311-345.

[7] Alvarado MC, Rudy JW. Rats with damage to the hippocampal-formation are impaired on the transverse-patterning problem but not on elemental discriminations. Behav Neurosci 1995;109(2):204-211.

[8] Alvarado MC, Rudy JW. A comparison of kainic acid plus colchicine and ibotenic acid-induced hippocampal formation damage on four configural tasks in rats. Behav Neurosci 1995;109(6):1052-1062.

[9] Dusek JA, Eichenbaum H. The hippocampus and transverse patterning guided by olfactory cues. Behav Neurosci 1998;112(4):762-771.

[10] Alvarado MC, Bachevalier J. Revisiting the Maturation of Medial Temporal Lobe Memory Functions in Primates. Learning Mem 2000;7(5):244-256.

[11] Alvarado MC, Wright AA, Bachevalier J. Object and spatial relational memory in adult rhesus monkeys is impaired by neonatal lesions of the hippocampal formation but not the amygdaloid complex. Hippocampus 2002;12(4):421-433.

[12] Rickard TC, Grafman J. Losing Their Configural Mind: Amnesic Patients Fail on Transverse Patterning. J Cogn Neurosci 1998;10(4):509-524. [13] Rickard TC, Verfaellie M, Grafman J. Transverse Patterning and Human Amnesia. J Cogn Neurosci 2006;18(10):1723-1733.

[14] Whishaw IQ, Tomie JA. Acquisition and retention by hippocampal rats 
of simple, conditional, and configural tasks using tactile and olfactory cues: Implications for hippocampal function. Behav Neurosci 1991;105(6):787-797. [15] Alvarado MC, Rudy JW. A comparison of "configural" discrimination problems: Implications for understanding the role of the hippocampal formation in learning and memory. Psychobiol 1995;23(3):178-184.

[16] McDonald RJ, Murphy RA, Guarraci FA, Gortler JR, White NM, Baker AG. Systematic comparison of the effects of hippocampal and fornix-fimbria lesions on acquisition of three configural discriminations. Hippocampus 1997;7(4):371-388.

[17] Meltzer JA, Negishi M, Constable RT. Biphasic hemodynamic responses influence deactivation and may mask activation in block-design fMRI paradigms. Hum Brain Mapp 2008;29(4):385-399.

[18] Meltzer JA, Fonzo GA, Constable RT. Transverse patterning dissociates human EEG theta power and hippocampal BOLD activation. Psychophysiology 2009;46(1):153-162.

[19] Mills T, Lalancette M, Moses SN, Taylor MJ, Quraan MA. Techniques for Detection and Localization of Weak Hippocampal and Medial Frontal Sources Using Beamformers in MEG. Brain Topogr 2012;25(3):248-263.

[20] Bruce CJ, Goldberg ME, Bushnell MC, Stanton GB. Primate Frontal Eye Fields. II. Physiological and Anatomical Correlates of Electrically Evoked Eye Movements. J Neurophysiol 1985;54(3):714-734.

[21] Inoue M, Mikami A. Feature to space conversion during target selection in the dorsolateral and ventrolateral prefrontal cortex of monkeys. Eur J Neurosci 2010;31(5):938-949.

[22] Asaad WF, Rainer G, Miller EK. Task-Specific Neural Activity in the Primate Prefrontal Cortex. J Neurophysiol 2000;84(1):451-459.

[23] Fuster JM, Bauer RH, Jervey JP. Cellular discharge in the dorsolateral prefrontal cortex of the monkey in cognitive tasks. Exp Neurol 1982;77(3):679-694.

[24] Funahashi S, Bruce CJ, Goldman-Rakic PS. Visuospatial Coding in Primate Prefrontal Neurons Revealed by Oculomotor Paradigms. J Neurophysiol 1990;63(4):814-831.

[25] Hoshi E, Shima K, Tanji J. Neuronal Activity in the Primate Prefrontal Cortex in the Process of Motor Selection Based on Two Behavioral Rules. J Neurophysiol 2000;83(4):2355-2373.

[26] Buckley MJ, Mansouri FA, Hoda H, Mahboubi M, Browning PGF, Kwok 
SC, Philllips A, Tanaka K. Dissociable Components of Rule-Guided Behavior Depend on Distinct Medial and Prefrontal Regions. Science 2009; 325(5936):52-58.

[27] Tsujimoto S, Sawaguchi T. Neuronal Representation of Response-Outcome in the Primate Prefrontal Cortex. Cereb Cortex 2004;14(1):47-55.

[28] Tsujimoto S, Sawaguchi T. Context-dependent Representation of Response-outcome in Monkey Prefrontal Neurons. Cereb Cortex 2005;15(7):888-898.

[29] Alvarado MC, Bachevalier J. Selective neurotoxic damage to the hippocampal formation impairs performance of the transverse patterning and location memory tasks in rhesus macaques. Hippocampus 2005;15(1):118-131.

[30] Alvarado MC, Bachevalier J. Comparison of the Effects of Damage to the Perirhinal and Parahippocampal Cortex on Transverse Patterning and Location Memory in Rhesus Macaques. J Neurosci 2005;25(6):1599-1609.

[31] Barbas H, Pandya DN. Architecture and intrinsic connections of the prefrontal cortex in the rhesus monkey. J Comp Neurol 1989;286(3):353-375. [32] Carmichael ST, Price JL. Connectional networks within the orbital and medial prefrontal cortex of macaque monkeys. J Comp Neurol 1996;371(2):179-207.

[33] Barbas H, Ghashghaei H, Dombrowski SM, Rempel-Clower NL. Medial prefrontal cortices are unified by common connections with superior temporal cortices and distinguished by input from memory-related areas in the rhesus monkey. J Comp Neurol 1999;410(3):343-367.

[34] Lavenex P, Suzuki WA, Amaral DG. Perirhinal and parahippocampal cortices of the macaque monkey: Projections to the neocortex. J Comp Neurol 2002;447(4):394-420.

[35] Kondo H, Saleem KS, Price JL. Differential connections of the perirhinal and parahippocampal cortex with the orbital and medial prefrontal networks in macaque monkeys. J Comp Neurol 2005;493(4):479-509.

[36] Hasegawa I, Fukushima T, Ihara T, Miyashita Y. Callosal Window Between Prefrontal Cortices: Cognitive Interaction to Retrieve Long-Term Memory. Science 1998;281(5378):841-818.

[37] Tomita H, Ohbayashi M, Nakahara K, Hasegawa I, Miyashita Y. Top-down signal from prefrontal cortex in executive control of memory 
retrieval. Nature 1999;401(6754):699-703.

[38] Miller EK, Cohen JD. An integrative theory of prefrontal cortex function. Annu Rev Neurosci 2001;24:167-202.

[39] Johnston K, Levin HM, Koval MJ, Everling S. Top-Down Control-Signal Dynamics in Anterior Cingulate and Prefrontal Cortex Neurons following Task Switching. Neuron 2007;53(3):453-462. 


\section{Figure captions}

Figure 1. Task paradigms.

(a) Sequences of task events in the TP (left) and DMS (right) tasks are depicted from top to bottom. Arrow heads in the saccade period indicate the target cue. Fixation 1, cue, saccade, hold, and prereward periods are common to both tasks. After the cue onset, both tasks progressed in the same way. (b) Stimulus sets for the 2 monkeys. (c) Combinations of the target and nontarget in the TP and DMS tasks. In each pair of cues, the target and nontarget are depicted in black and gray, respectively. In the congruent trials of the DMS task, the assignment of the target is the same as in the TP task.

Figure 2. Recording sites.

(a) Location of the recording grid (gray unfilled circles) on the line drawing of the brain of monkey $\mathrm{C}$. Three black dots indicate the locations of pin holes made at the time of perfusion. The arrow head indicates the location of the pin hole shown in (c). Black arrow indicates the location of an electrolytic lesion depicted in (b). (b) Photograph of a section through an electrolytic lesion [location indicated in (a)]. (c) A coronal section through the track of a pin penetration [location indicated by a black arrowhead in (a)]. (d) Recording sites were divided into 3 regions (PS, DC, and MPF) as illustrated on a coronal section of the brain. $C g S$, cingulate sulcus; $P S$, principal sulcus; $s A S$, superior limb of arcuate sulcus.

Figure 3. Neuronal responsiveness in the TP task.

Raster displays and spike density function of a C-type neuron (a), a P-type neuron (b), and a CP-type neuron (c). Shading in each panel indicates the analysis period. Panels on the far left show the activities in the control period. The activities are aligned at the fixation onset (ordinate). Second-left panels show the activities in the cue period. The activities are aligned at the choice cue onset (thin vertical line). Third-left panels show the activities in the presaccadic period. The activities are aligned at the saccade onset (thin vertical line). In the right panels, cue period responses to different shapes (a, c) and presaccadic period responses to different locations (b) are depicted. A, $\mathrm{B}, \mathrm{C}$ indicate the shapes, whereas $\mathrm{L}$ and $\mathrm{R}$ indicate the locations of the target. CON, control period; CUE, cue period; PRE, presaccadic period. 
Figure 4. Effects of target shape, target location, and task type on the neuronal response.

Raster displays and spike density functions of 4 representative neurons showing (a) task dependency in the cue period, (b) task and location dependency in the cue period, (c) task and location dependency in the presaccadic period, and $(\mathrm{d})$ shape and location dependency in the presaccadic period. Left, middle, and right panels indicate the responses to different target shapes (A, B, and C), different target locations (left and right), and different task types (TP and DMS), respectively. Other conventions are as in Fig. 3.

Figure 5. Task interaction neurons

Neuronal responses of 2 representative "task interaction" neurons are displayed separately for the TP (left) and DMS (right) tasks. (a) Cue period responses for different target shapes (A, B, C) are indicated separately. (b) Presaccadic period responses for different combinations of shapes (A, B, C) and locations $(\mathrm{L}, \mathrm{R})$ are indicated separately. Other conventions are as in Figs. 3 and 4.

Figure 6. Task-dependent and location-dependent postsaccadic activities Raster displays and spike density functions aligned at the saccade offset (thin vertical line). The shadings indicate the postsaccadic period. (a) A neuron showing differential response after the TP and DMS tasks. (b) A neuron showing differential response after the saccade to the left and right target.

Figure 7. Postsaccadic responses in the correct and erroneous trials

Postsaccadic responses of 2 neurons (a, b) in the correct (left) and erroneous (right) trials. The neuron in (a) showed similar location dependency after both the correct and the erroneous trials. The neuron in (b) showed a location dependency in the correct trials, whereas it showed vigorous response in erroneous trials regardless of the location. Conventions are as in Fig. 6.

Figure 8. Task-dependent activities starting in the fixation period Left, middle, and right panels show the activities in the control, cue, and presaccadic periods, respectively. Neuronal responses to the TP and DMS 
tasks are shown separately in each panel. The neuron in (a) showed a higher activity for the DMS task until the cue period. The neuron in (b) showed a higher activity for the TP task throughout the trial. Conventions are as in Figs. 3 and 4.

Figure S1. Distribution of the TP task responsive neuron of monkey C Recording sites in 12 successive 100- $\mu$ m-thick sections were superimposed on the drawing of the representative section. Blue-filled, red-filled, yellow-filled, and black-filled circles indicate the recording sites of the C-type, P-type, CP-type neurons, and unclassified responsive neurons, respectively. Unfilled circles show the recording sites of the nonresponsive neurons.

Figure S2. Distribution of the TP task responsive neuron of monkey M Recording sites in 9 successive 100- $\mu \mathrm{m}$-thick sections were superimposed on the drawing of the representative section. Other conventions are the same as in Fig. S1 


\section{Figure 1}

a

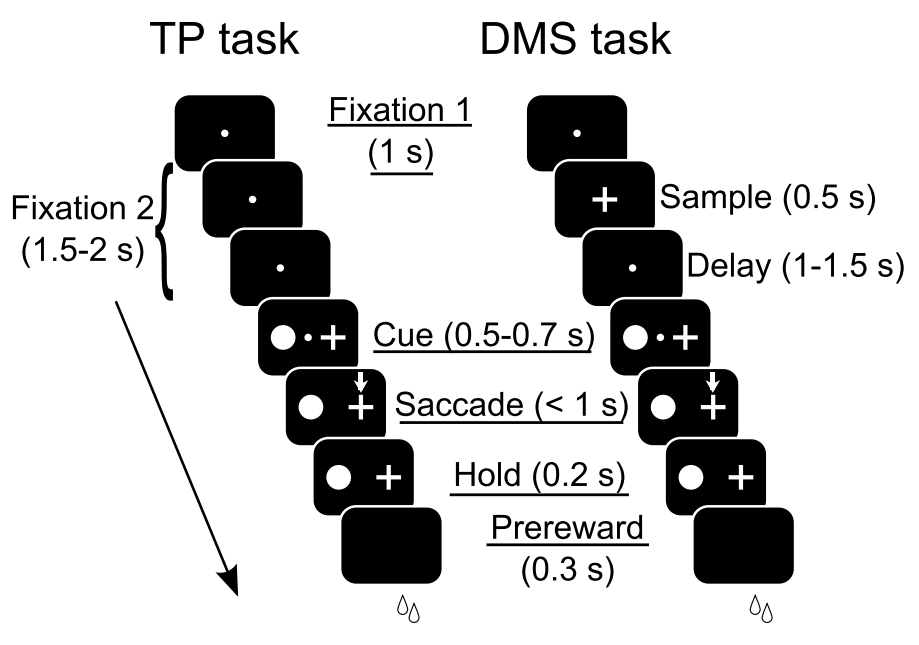

b

\begin{tabular}{llll} 
Stimulus & A & B & C \\
\hline Monkey C & + & $\mathbf{O}$ & $\boldsymbol{\Delta}$ \\
\hline Monkey M & $\diamond$ & $\boldsymbol{B}$ & $\star$
\end{tabular}

C TP task DMS task Congruent Incongruent

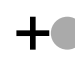

$+$

$+$

$+$

-

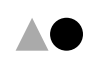

$\mathbf{\Delta +}$

$+\mathbf{\Delta}$

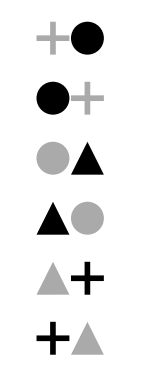


a

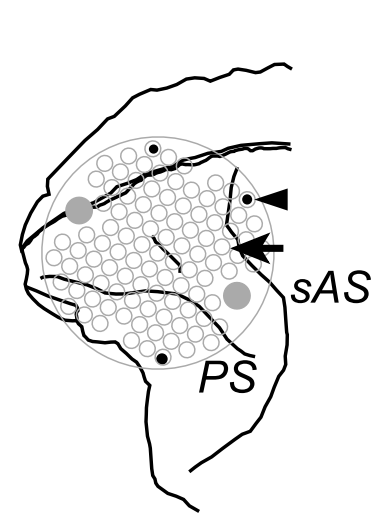

C

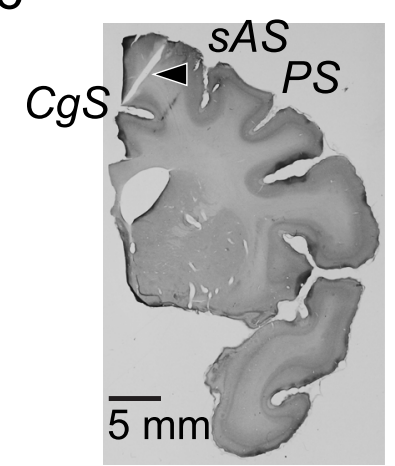

b

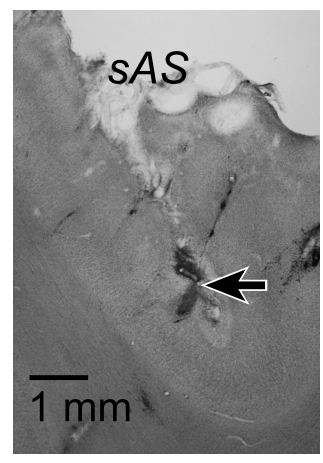

d

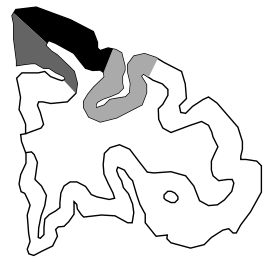

PS

DC

MPF 


\section{Figure 3}

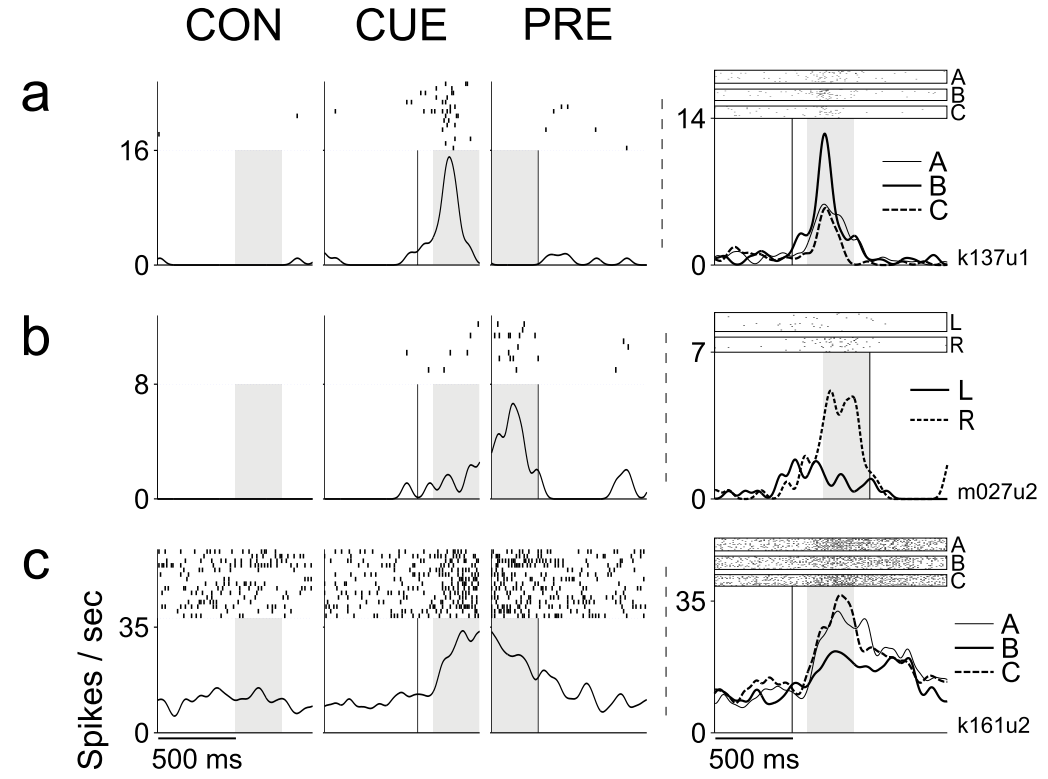




\section{Figure 4}

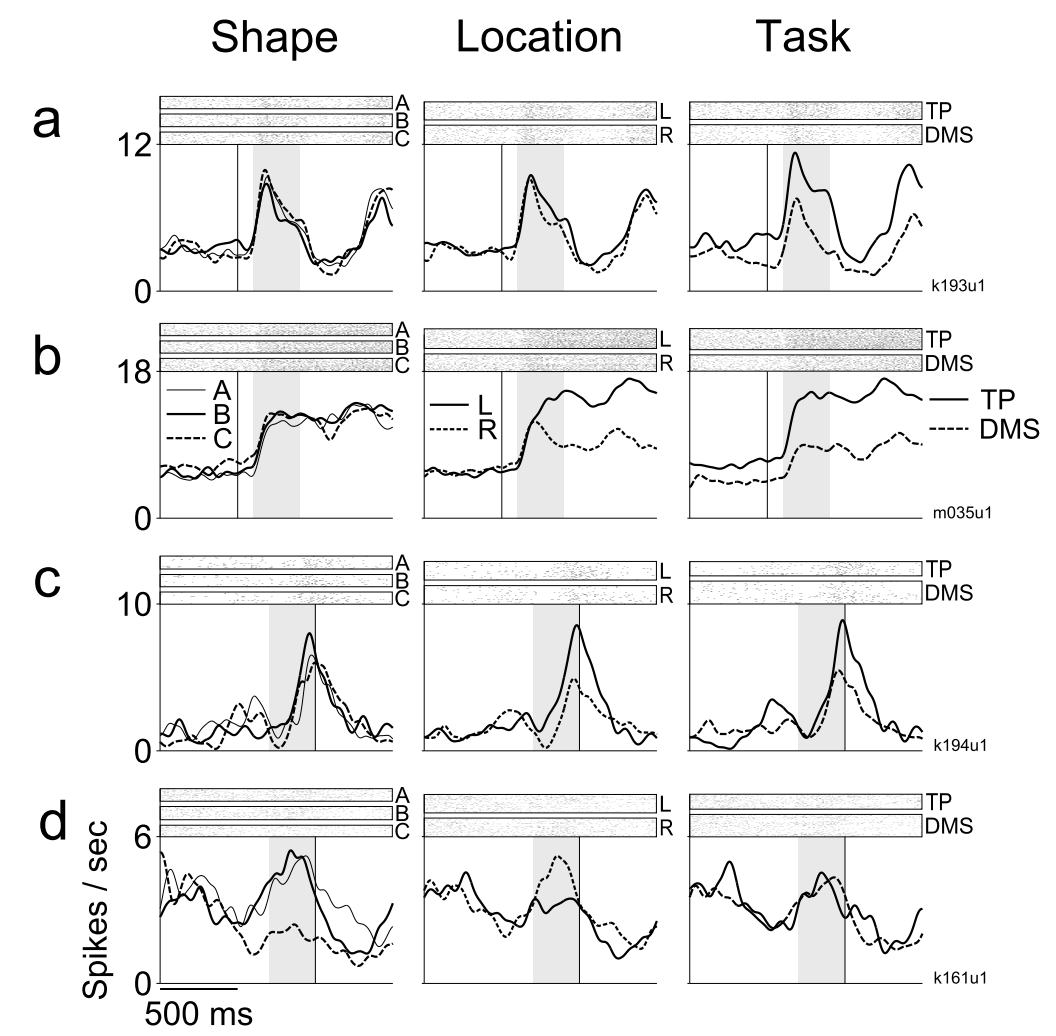




\section{TP DMS}

a

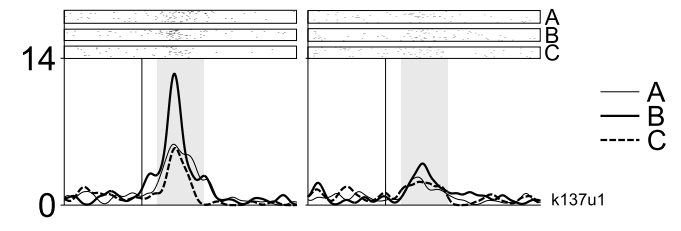

b

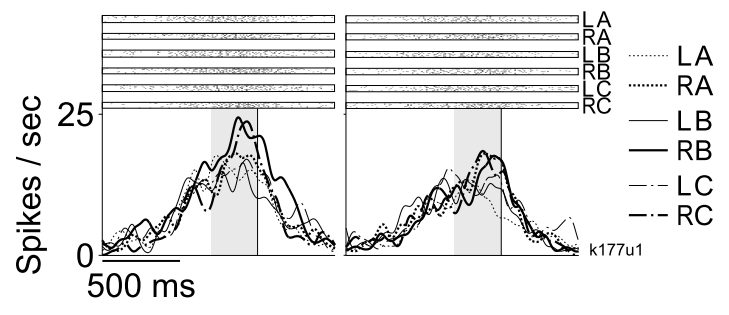


Figure 6

a

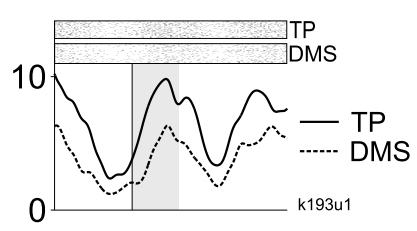

b

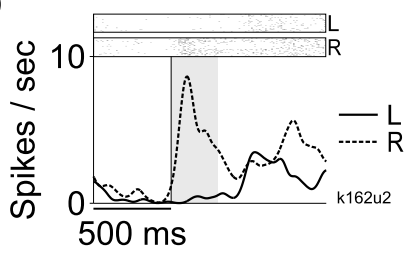




\section{Figure 7}

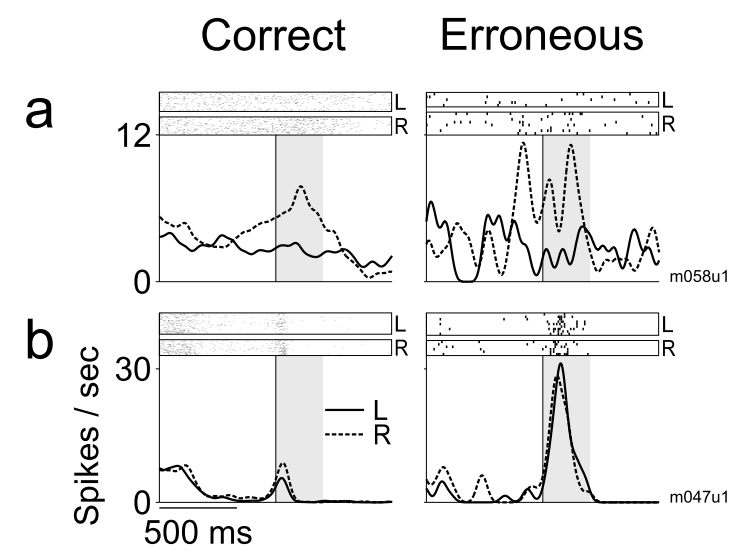


Figure 8

\section{CON CUE PRE}

a

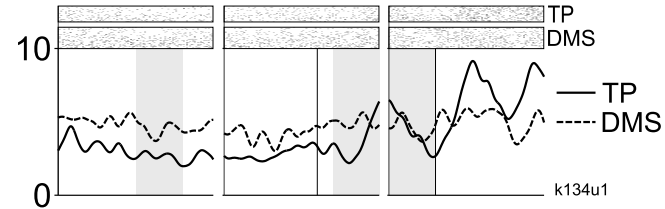

b

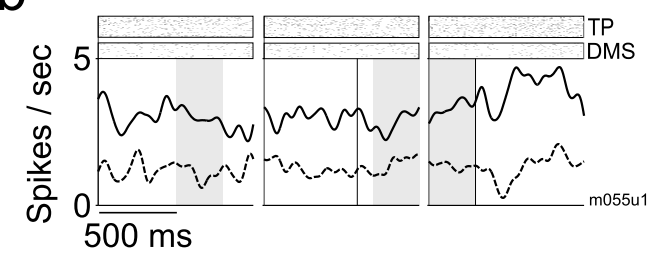


Table 1. Selectivity for the shape, location, and task

\begin{tabular}{lllllll}
\hline \multicolumn{5}{c}{ Cue period } & \multicolumn{3}{c}{ Presaccadic period } \\
& PS & DC & MPF & PS & DC & MPF \\
\hline Main effect & & & & & \\
S & $6(9 \%)$ & $2(3 \%)$ & $6(11 \%)$ & $12(19 \%)$ & $5(7 \%)$ & $6(11 \%)$ \\
L & $7(11 \%)$ & $4(6 \%)$ & $6(11 \%)$ & $22(34 \%)$ & $8(11 \%)$ & $17(32 \%)$ \\
T & $20(31 \%)$ & $20(28 \%)$ & $28(52 \%)$ & $23(36 \%)$ & $27(38 \%)$ & $24(44 \%)$ \\
& & & & & \\
Combinations of the main effects & & & & \\
S only & $2(3 \%)$ & $1(1 \%)$ & $1(2 \%)$ & $6(9 \%)$ & $2(3 \%)$ & 0 \\
L only & $4(6 \%)$ & $4(6 \%)$ & $1(2 \%)$ & $12(19 \%)$ & $5(7 \%)$ & $6(11 \%)$ \\
T only & $15(23 \%)$ & $19(26 \%)$ & $20(37 \%)$ & $13(20 \%)$ & $21(29 \%)$ & $12(22 \%)$ \\
S + L & $1(2 \%)$ & 0 & $1(2 \%)$ & $2(3 \%)$ & 0 & $1(2 \%)$ \\
S + L + T & 0 & 0 & 0 & $2(3 \%)$ & 0 & $3(6 \%)$ \\
S + T & $3(5 \%)$ & $1(1 \%)$ & $4(7 \%)$ & $2(3 \%)$ & $3(4 \%)$ & $2(4 \%)$ \\
L + T & $2(3 \%)$ & 0 & $4(7 \%)$ & $6(9 \%)$ & $3(4 \%)$ & $7(13 \%)$ \\
\hline
\end{tabular}

Three-way ANOVA was applied to 64 neurons in PS, 72 in DC, and 54 in MPF. Abbreviation: S, shape; L, location; T, task. 
Table 2. Numbers of neurons showing differential activity between TP and DMS tasks

\begin{tabular}{lllllll}
\hline & \multicolumn{2}{l}{ Cue period } & \multicolumn{3}{c}{ Presaccadic period } \\
& PS & DC & MPF & PS & DC & MPF \\
\hline TP > DMS & 12 & 12 & 15 & 18 & 11 & 14 \\
DMS $>$ TP & 8 & 8 & 13 & 5 & 16 & 10 \\
\hline
\end{tabular}

Numbers of neurons exhibiting significantly higher firing rate in the TP task (TP > DMS) and in the DMS task (DMS > TP). 
We first trained the monkeys on the DMS task. The training on the TP task started after each monkey reached the 70\% correct in the DMS task. The monkeys performed 1-3 blocks per task in each daily session. The training procedure for the TP task conformed to the previous studies [29,30]. Briefly, the monkeys were first trained on the discrimination of a single stimulus pair. After the correct rate reached a criterion (90\%), a second pair was introduced. If the correct rates for both stimulus pairs reached the criterion, the third pair was added. The monkey $\mathrm{C}$ needed 3, 5, and 149 sessions to achieve the criterion in the 1st, 2nd, and 3rd discriminations, respectively. Similarly, the monkey $\mathrm{M}$ spent 5, 7, and 77 sessions to meet the criterion in the 1st, 2nd, and 3rd discriminations, respectively. We started the recording of the neuronal activities after the monkey reached the $90 \%$ correct in both tasks. 
Supplementary data 2. Comparison of neuronal activities among the TP task, congruent, and incongruent trials of the DMS task

The task-dependent neurons could also change the activity between the congruent and incongruent trials of the DMS task. To test this possibility, we compared the neuronal firing of the task-dependent neurons, in the cue and presaccadic periods, among the trials of the TP task, congruent, and incongruent trials of the DMS task using a Tukey's HSD test $(\alpha=0.05)$ as a multiple comparison test.

Among the 68 and 74 neurons showing the task-dependency during the cue and presaccadic periods, 66 (97\%) and 70 (95\%), respectively, showed indistinguishable firing between the congruent and incongruent trials. The numbers were not significantly different among the three prefrontal regions (cue period, $\chi^{2}=0.94, P>$ 0.05; presaccadic period, $\chi^{2}=0.71, P>0.05$, Fisher's exact test). Additionally, among the 122 and 116 task-independent neurons during the cue and presaccadic periods, 121 (99\%) and 112 (97\%), respectively, showed indistinguishable firing between the congruent and incongruent trials. These numbers were also not significantly different among the three prefrontal regions (cue period, $\chi^{2}=0.53, P>0.05$; presaccadic period, $\chi^{2}=0.95, P>0.05$, Fisher's exact test). 


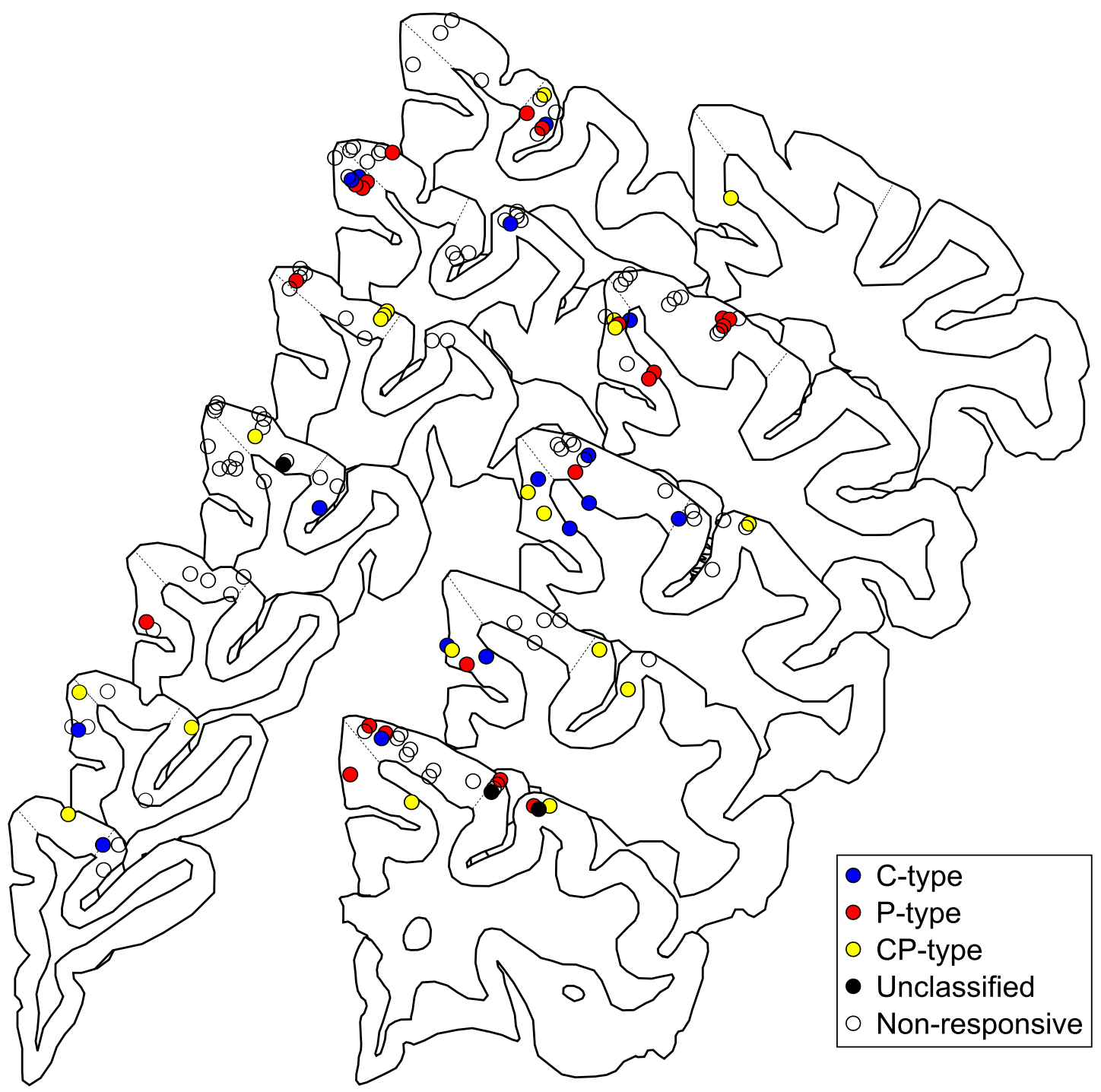

Fig. S1. Distribution of the TP task responsive neuron of monkey $\mathrm{C}$

Recording sites in 12 successive 100- $\mu$ m-thick sections were superimposed on the drawing of the representative section. Blue-filled, red-filled, yellow-filled, and black-filled circles indicate the recording sites of the C-type, P-type, CP-type neurons, and unclassified responsive neurons, respectively. Unfilled circles show the recording sites of the nonresponsive neurons. 


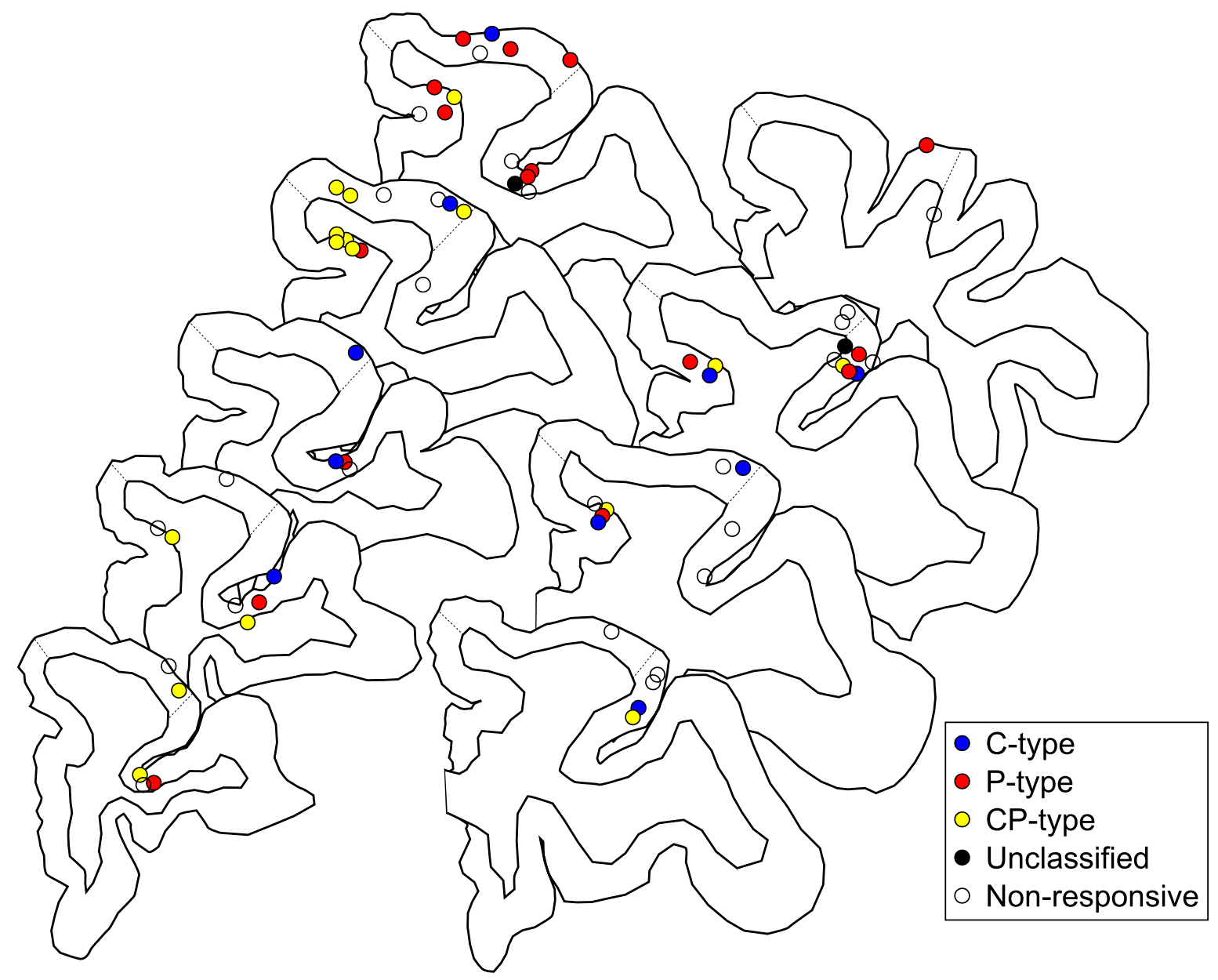

Fig. S2. Distribution of the TP task responsive neuron of monkey M Recording sites in 9 successive $100-\mu m$-thick sections were superimposed on the drawing of the representative section. Other conventions are the same as in Fig. S1 